\title{
RADICAL EXTENSIONS OF RINGS
}

\author{
CARL FAITH ${ }^{1}$
}

Jacobson's generalization [ 5 , Theorem 8$]$ of Wedderburn's theorem [8] states that an algebraic division algebra over a finite field is commutative. These algebras have the property that some power ${ }^{2}$ of each element lies in the center. Kaplansky observed in [7] that any division ring, or, more generally, any semisimple ring, in which some power of each element lies in the center is commutative. Kaplansky's idea was generalized in [1], and radical extensions of arbitrary subrings were studied, where the ring $A$ is a radical extension of the ring $B$ in case each $a \in A$ is radical over $B$ in the sense that some power of $a$ lies in $B$. In this connection Theorem A of [1] states:

If $A$ is a simple ring with a minimal one-sided ideal, and radical over a subring $B \neq A$, then $A$ is a field.

This is the best possible result of this type for which $A / B$ is radical, and no restriction is placed on $B$ (best in the sense that there exist noncommutative primitive rings with minimal one-sided ideals and radical over proper simple subrings $[1, \S 4])$. The starting point for the present investigation is the observation that if $A$ is a division ring and radical over center, then $A$ is a radical extension of both a division subring, and a commutative subring. Accordingly, the present paper is devoted to the study of rings $A$ which are radical over (1) division subrings, or (2) commutative subrings. In connection with (1), there exists the following generalization of the WedderburnJacobson-Kaplansky theorems on division rings.

TheOREM 1.1. If $A$ is a ring with no nil ideals $\neq\{0\}$, and if $A$ is radical over a division subring $B \neq A$, then $A$ is a field.

Corresponding to (2), one has the following extension of Kaplansky's theorem for semisimple rings. In it (and throughout this paper) $J(A)$ denotes the Jacobson radical of the ring $A$.

THEOREM 1.2. If $A$ is radical over a commutative subring $B$, and if $J(A)=\{0\}$, then $A$ is commutative.

Kaplansky's theorem [7] is, then, the special case of Theorem 1.2 for which $B$ is central.

Received by the editors September 10, 1959, and in revised form, April 30, 1960.

1 The author presently is North Atlantic Treaty Organization (U.S.A.) Postdoctoral Fellow in Heidelberg University.

2 Here, and below, the "zero power" is excluded. 
In $\$ 2$ necessary and sufficient conditions are given in order that a ring $A$ be a radical extension of a division ring $B$. These conditions are strong enough to enable one to deduce that the only radical extensions of a division ring $B$, when $B$ is either noncommutative, or has characteristic 0 , are the trivial ones: $B \oplus N$, where $N$ is an arbitrary nil ring.

In $\S 3$ under the hypotheses (i) $J(A) \neq A$ and (ii) $A$ has no nil ideals $\neq\{0\}$, it is shown that in order that $A$ be a radical extension of a simple subring $B$ it is necessary that $A$ be a primitive ring. Then, if $B$ contains an identity, $A$ itself is simple. Moreover, in order that $A$ be primitive (resp. semisimple) and a radical extension of a $\operatorname{ring} B$, it is necessary that $B$ itself be primitive (resp. semisimple; cf. Theorem $3.2 \mathrm{ff}$ ).

$\$ 4$ contains an application of Theorem 1.1 on the structure of the multiplicative group of a division ring.

1. This section contains proofs of Theorems 1.1 and 1.2, and several results related to theorems of Herstein.

Proof of Theorem 1.1. Since $A$ is not a radical ring in the sense of Jacobson [6], that is, since the Jacobson radical $J(A) \neq A, A$ contains a primitive ideal $P$. If $P \cap B \neq\{0\}$, then $P \supseteq B$ and $A-P$ would be a nil primitive ring. This contradiction shows that $P \cap B=\{0\}$. Then $P$, being a radical extension of $\{0\}=P \cap B$, is nil. By hypothesis, $P=\{0\}$, so that $A$ is a primitive ring. Similarly, since $A$ contains no nil left ideals, if $Q$ is any nonzero left ideal in $A$, necessarily $Q \supseteq B$. In particular, $B$ is contained in the intersection of the nonzero modular maximal left ideals of $A$. Since the intersection of all of the modular maximal left ideals is $J(A)$, and since $J(A)=\{0\}$, it follows that $\{0\}$ is a modular maximal ideal, so that $A$ is a division ring. Then $A$, being a division ring which is radical over a division subring $B \neq A$, is a field, according to [1, Theorem A].

Proof of Theorem 1.2. The primitive homomorphic images of $A$ inherit the hypotheses on $A$. Since $A$ is a subdirect sum of primitive rings, and since a subdirect sum of commutative rings is itself commutative, it suffices to consider the case where $A$ is primitive. Assume that $A$ is a noncommutative primitive ring. Then, by Kaplansky's theorem, $B$ is not contained in the center $Z$ of $A$. By [1, Theorem A], $A$ cannot be a division ring. Thus, $A$ has a representation as a dense ring of linear transformations on a vector space $V$ of dimension $>1$ over a division ring. Choose $b \in B, b \notin Z$. Then, there exists $x \in V$ such that $x$ and $x b$ are linearly independent. By the density of $A$, there exists $a \in A$ mapping both $x$ and $x b$ onto $x$. Then, for this $a$, no power of $a$ commutes with $b$. Then, since $B$ is commutative, no 
power of $a$ can lie in $B$. Thus, $A / B$ is not radical, a contradiction which proves the theorem.

Theorem 1.2 has the following corollaries.

CoROLlary 1.3. If $A$ is a noncommutative semisimple ring, and if $a \in A$ is noncentral, then there exists $b \in A$ such that $b^{n} a \neq a b^{n}$, for all $n \geqq 1$.

Proof. Let $a \in A$ be noncentral, and let $a^{\prime}$ denote the corresponding element in the primitive homomorphic image $A^{\prime}$ of $A$. For some $A^{\prime}, a^{\prime}$ is noncentral. Then in this $A^{\prime}$ there exists, by the proof of the theorem, $b^{\prime}$ such that no power of $b^{\prime}$ commutes with $a^{\prime}$. Then if $b \in A$ maps onto $b^{\prime}$ under the homomorphism $A \rightarrow A^{\prime}$, then no power of $b$ commutes with $a$.

Corollary 1.4. If $A$ is semisimple, and if $N$ is a fixed natural number such that to each pair $x, y \in A, x$ is radical over the centralizer of $y^{N}$ in $A$, then $A$ is commutative.

Proof. If $A$ were noncommutative, then, by Kaplansky's theorem, there would exist $y \in A$ such that $y^{N}$ is noncentral. Then $A$ is not radical over the centralizer of $y^{N}$ by the corollary. This contradiction completes the proof.

In connection with the last corollary, it is of interest to ask what conditions on $A$ imply commutativity, if $A$ satisfies:

(*) For every $x, y \in A$, there exist natural numbers $n, m$ such that $x^{n} y^{m}=y^{m} x^{n}$.

Since any ring $A$ radical over a commutative subring $B$ satisfies (*), in view of Theorem 1.2 it is natural to ask if every semisimple ring satisfying (*) is commutative. The next theorem gives a partial answer.

THEOREM 1.5. If $A$ is a primitive ring with a minimal left ideal, or if $A$ is a subdirect sum of such rings, and if $A$ satisfies (*), then $A$ is commutative.

Proof. It suffices to assume that $A$ is primitive, and even further that $A$ coincides with its socle, that is, that $A$ is simple with a minimal left ideal. If $y \in A$, then

$$
(y)^{\prime} \subseteq\left(y^{2}\right)^{\prime} \subseteq\left(y^{6}\right)^{\prime} \subseteq \cdots \subseteq\left(y^{n !}\right)^{\prime} \subseteq \cdots,
$$

where as before the primes denote centralizers in $A$. Clearly (*) implies that $A$ is radical over $B_{y}=\cup_{1}^{\infty}\left(y^{n !}\right)^{\prime}$, so by $[1$, Theorem A] noncommutativity of $A$ implies equality $A=B_{y}$ for each $y \in A$. Thus, if 
$x \in A$, then for each $y \in A, x \in\left(y^{m}\right)^{\prime}$, that is, $y^{m} \in x^{\prime}$, for suitable $m$, so that $A$ is radical over $x^{\prime}$ for each $x \in A$. Then by Corollary 1.4 commutativity of $A$ follows, a contradiction which proves the theorem. Herstein's extension [3] of Kaplansky's theorem states that a ring $A$ containing no nil ideals $\neq\{0\}$, and radical over center is commutative. This raises the following question which is left open:

If $A$ is a ring with no nil ideals $\neq\{0\}$ and radical over a commutative (possibly semisimple) subring $B$, is $A$ necessarily commutative?

If $B$ is a semisimple ideal, the answer is yes. This can be seen as follows: $J(B)=\{0\}$ so that $J(A) \cap B=\{0\}$, whence $J(A)$ is nil. By hypothesis, $J(A)=\{0\}$, and Theorem 1.2 applies.

In view of Herstein's [4, Theorem 1] one might ask if any ring $A$ with no nil ideals $\neq\{0\}$, and satisfying (*) is commutative. The question is open even if $m$ is fixed as in Corollary 1.4.

2. The main purpose of this section is to present a characterization of non-semisimple rings which are radical over division rings. The structure theory for such rings can be reduced to the ones which are directly irreducible. An example of a radical extension of a division ring which is noncommutative, non-semisimple, and directly irreducible is given.

Lemma 2.1. Let $A$ be a ring with a subring $B$ such that $B$ contains an identity $e$, and such that $A / B$ is radical. Then

$$
A=N \oplus Q,
$$

where $N=(1-e) A(1-e)$ is a nil ideal, and $Q=e A e$.

Proof. Write $A=e A e+f A f+e A f+f A e$, where (formally) $f=1-e$, and let $u \in e A f$. Since $(e+u)^{n}=e+u$, for all $n, e+u \in B \subseteq e A e$. Then, $0=u \in e A e \cap e A f=\{0\}$, that is, $e A f=\{0\}$. By symmetry, $f A e=\{0\}$, so that $A=e A e \oplus f A f$. Clearly, $f A f$, being radical over $e A e \cap f A f=\{0\}$, is nil.

THEOREM 2.2. In order that a ring $Q$ be a radical extension of a subring $B$, the following three conditions are sufficient:

$(\alpha) Q$ contains a nil ideal $I$ such that $Q$ is radical over $B+I$;

$(\beta)$ for all $i \in I, B$ is radical over $B \cap i^{\prime}$, where $i^{\prime}$ is the centralizer of $i$ in $Q$;

( $\gamma$ ) $Q$ has prime characteristic $p$.

Proof. Let $a \in Q$, and, by $(\alpha)$, choose $m$ such that $a^{m} \in B+I$, $a^{m}=b+i, b \in B, i \in I$. By $(\beta)$, choose $N$ such that $b^{N} i=i b^{N}$, and write $a^{m N}=b^{N}+j$, where $j \in I$. Since $b^{N}$ commutes with $a^{m}, b^{N}$ commutes 
with $a^{m N}$, so that $b^{N}$ commutes with $j=a^{m N}-b^{N}$. Then if $p^{f} \geqq$ the index of $j$,

$$
a^{m N p^{f}}=b^{N p^{f}} \in B
$$

as desired.

THEOREM 2.3. The radical extensions of a division ring $B$ are the direct sums

$$
Q \oplus N,
$$

where $N$ is an arbitrary nil ring, and $Q$ is a directly irreducible radical extension of $B$. Necessary and sufficient conditions for a directly irreducible ring $Q \supset B$ (and $Q \neq B$ ) to be a radical extension of $B$ are as follows:

(1) The identity e of $B$ is the identity of $Q ; J=J(Q)$ is a nil ideal; and $Q-J$ is a field which is radical over $(B+J)-J$.

(2) $B$ is a field, and for all $q \in B+J, Q$ is radical over $B \cap q^{\prime}$, where $q^{\prime}$ is the centralizer in $Q$ of $q$.

(3) $Q$ has prime characteristic. ${ }^{3}$

Proof. If $A$ is a radical extension of $B$, then by Lemma 2.1, $A=Q \oplus N$, where $Q=e A e$, and $N=(1-e) A(1-e)$ is nil. Suppose that $Q=L \oplus K$, where $L$ and $K$ are ideals of $Q$. Both $L /(L \cap B)$ and $K /(K \cap B)$ are radical extensions. Since $Q$ is non-nil, it can be assumed that $L \cap B \neq\{0\}$. Then, since $B \subseteq L, e \in L$, so that $L=Q$ and $K=\{0\}$, that is, $Q$ is directly irreducible. Conversely, $Q \oplus N, Q$ and $N$ as above, are radical extensions of $B$.

(1) From now on, assume that $Q \neq B$. Since $J \cap B=\{0\}, J$ is nil; $Q-J$ is a field by Theorem 1.1, if $Q \neq B+J$. If $Q=B+J$, that $Q-J$ is a field follows from (2), so that the proof of (1) is reduced to that of (2). Furthermore, if $J=\{0\}$, then $Q$ is a field by Theorem 1.1, since then $Q$ is a ring with no nil ideals $\neq\{0\}$, and is a proper radical extension of $B$. Thus, in the case $J=\{0\},(2)$ is trivial, and (3) follows from Kaplansky's lemma [7]. Hence, only the $J \neq\{0\}$ case of (2) and (3) remains.

(2) The verbatim proof of [1, Lemma 1] yields the following result which is needed below. ${ }^{4}$ (The notation is that of [1].)

Let $D$ be a ring with identity 1 , and let $\Delta$ be a division subring $\neq D$ such that $D / \Delta$ is radical. Let $v \in D, v \notin \Delta$ be such that $\left(v+c_{i}\right)^{-1}$ exists

${ }^{3}$ Considerably more information can be squeezed out of the hypotheses. See, for example, a following article of mine [2], and Kaplansky's lemma [7].

4 This result is extensively generalized in [2] in a situation where arbitrary polynomials play the role of the nomomials $x^{n}$. Nevertheless, even the proof of this more general result is little more than a rewriting of the proof of [1, Lemma 1]. 
for different $c_{i} \in \Delta, i=1$ and 2 . Then, if $d \in \Delta$ commutes with both $c_{1}$ and $c_{2}$, then $d^{n} v=v d^{n}$, for some $n \geqq 1$.

Choose $0 \neq j \in J$, and $b \in B$. Since $(j+c)^{-1}$ exists for all (more than one) nonzero $c \in B \cap b^{\prime}$, by the result just announced, $b$ is radical over $j^{\prime}$. By setting $v=j+d, 0 \neq j \in J, 0 \neq d \in B$, it follows in the same way that $b$ is radical over $(j+d)^{\prime}$. These two statements together show that $B /\left(B \cap q^{\prime}\right)$ is radical, for all $q=j+d \in J+B$. Since $Q / B$ is radical, so is $Q /\left(B \cap q^{\prime}\right)$. If $B=B \cap b^{\prime}$ for all $b \in B$, then $B$ is commutative. Otherwise, $B$ is radical over a proper subring, and $B$ is commutative by $[1$, Theorem A or B]. This completes the proof of (2).

(3) Let $0 \neq j \in J$ have index of nilpotency $m \geqq 2$, and let $n$ be the minimal natural number for which

$$
(e+j)^{n}=e+n j+\cdots+j^{n} \in B .
$$

Then,

$$
b=n j+\cdots+j^{n} \in B \cap J=\{0\} .
$$

If $m=2$, then $b=n j=0$. If $m>2$, then $b j^{m-2}=n j^{m-1}=0$. Hence, in either case, $n j^{m-1}=0$, showing that $n e=0$. Since $n \neq 0, B$ has prime characteristic which divides $n$. Then $Q=e Q e$ also has prime characteristic.

The sufficiency of (1)-(3) follows from Theorem 2.2.

REMARK. Theorem 1.1 is contained in the statement of Theorem 2.3.

The following. corollary is immediate.

COROLlaRY 2.4. If $B$ is a division ring which is noncommutative, or has characteristic 0 , then the radical extensions of $B$ are the direct sums $B \oplus N$, where $N$ is an arbitrary nil ring.

ExAmple. Below is an example illustrating Theorem 2.3 in the case $J(Q) \neq\{0\}$ and $Q=e A e$ is directly irreducible (as before, $e$ is the identity of $B$ ). Let $Q$ be the algebra of four dimensions over $G F(2)$ with a basis $e, \alpha, j, \alpha j$, and the table:

\begin{tabular}{c|c|c|c|c|} 
& $e$ & $\alpha$ & $j$ & $\alpha j$ \\
\hline$e$ & $e$ & $\alpha$ & $j$ & $\alpha j$ \\
\hline$\alpha$ & $\alpha$ & $\alpha-e$ & $\alpha j$ & $(\alpha-e) j$ \\
\hline$j$ & $j$ & $(\alpha-e) j$ & 0 & 0 \\
\hline$\alpha j$ & $\alpha j$ & $(\alpha-e) j$ & 0 & 0 \\
\hline
\end{tabular}


One easily verifies: (1) $Q$ is associative; (2) $J(Q)=\{0, j\}$; and (3) $B=(0, e, \alpha, \alpha-e) \cong G F(4)$. Since $Q$ satisfies the conditions of Theorem $2.2, Q / B$ is radical. Also one may verify this directly, since if $x \in Q$, then $x=a+b j$, where $a, b \in B$, and

$$
x^{6}=\left[(a+b j)^{3}\right]^{2}=[e+i]^{2}=e \in B,
$$

where $a \neq 0$ (where $i \in J$ ), and $x^{2}=0$, when $a=0$. (Note that $B \cong G F(4)$ is radical over $G F(2)$, a fact predicted by Theorem 2.3 , since $J$ does not lie in the centralizer of $B$ in $Q$.)

3. As I mentioned in the introduction, [1] contains examples of noncommutative, nonsimple primitive rings (of arbitrary characteristic) which are radical over their socles, that is, over simple subrings, a fact which precludes the existence of theorems of type 1.1 for radical extensions of simple rings. Nevertheless, simplicity of $B$ has noteworthy implications for radical extensions of $B$, as the following theorem indicates.

Theorem 3.1. If $A$ is a ring with no nil ideals $\neq\{0\}$, and if $A$ is a radical extension of a simple ring $B, A$ not a radical ring, then $A$ is a primitive ring.

Proof. If $I$ is any nonzero ideal of $A$, since $I$ is non-nil, then $I \cap B \neq\{0\}$, so that $I \supseteqq B$, by simplicity of $B$. Now $B \Phi J(A)$, since, otherwise, $A-J(A)$ would be a nil ring, and, then, $A=J(A)$, contrary to the hypothesis. Hence, $J(A)=\{0\}$, and $A$ is primitive in the same way as the proof of Theorem 1.1.

CoROLlaRy. If $A$ is a ring with no nil ideals $\neq\{0\}$, if $A$ is a radical extension of a simple ring $B$, and if $B$ contains an identity $e$, then $A$ is a simple ring with identity $e .^{5}$

Proof. Clearly, $A$ is not a radical ring, so that $A$ is primitive by the theorem. By Lemma 2.1, $A=e A e$, so that $e$ is an identity element in $A$. The first line in the proof of the theorem shows: If $I$ is any nonzero ideal of $A$, then $I \supseteqq B$. Then $e \in I$, so that $I=A$ is simple.

Now let $A$ be a noncommutative primitive ring, and let $B$ be a subring such that $A / B$ is radical. Represent $A$ as a dense ring of 1.t.'s in a vector space $V$ over a division ring $D$. Since $A$ is noncom-

b It is not known whether there exists a noncommutative simple ring which is radical over a subring $B$ (even if one assumes that $B$ is simple, and that $A$ contains an identity element $e$ ). As I pointed out in [1], a proof of the nonexistence of such rings (for which the parenthetical hypotheses are omitted) will preclude the existence of non-nilpotent simple nil rings, since these are necessarily noncommutative and radical over every subring. 
mutative, $A$ is not a division ring, and so $\operatorname{dim} V>1$. I wish to show that $B$ is isomorphic to a dense ring of 1.t.'s in $V$. It suffices to show, if $V_{n}$ is vector subspace of $V$ of finite dimension $n$, that every.1.t. of $V_{n}$ induced by an element of $A$ can be induced by an element of $B$, $n=1,2, \cdots$. (Then, every 1.t. in $V_{n}$ can be induced by an element of $B$.) Accordingly, let $U=\left\{a \in A \mid V_{n} a \subseteq V_{n}\right\}$, and $K=\left\{a \in A \mid V_{n} a=\{0\}\right\}$. By the density of $A$, if $\bar{U}$ is the difference ring $U-K$, then $\bar{U} \cong D_{n}$, the complete ring of $n \times n$ matrices over $D$. Since $U$ is radical over $Q=U \cap B$, then $\bar{U}$ is radical over $\bar{Q}$. First suppose $n>1$. Then, by $[1$, Theorem A], $\bar{U}=\bar{Q}$, that is, $U=Q+K$. This shows, if $\bar{a}$ is any 1.t. in $V_{n}$ which is induced by $a$ in $A$, then $a \in U$ so that $a=b+k$, with $b \in Q \subseteq B, k \in K$. Then $b \in B$ induces $\bar{a}$ as well. The case $n=1$ remains. Now $V_{1}$ is contained in a 2-dimensional subspace $V_{2}$, and if $\bar{a}_{1}$ is any 1.t. in $V_{1}$, there exists a 1.t. $\bar{a}_{2}$ in $V_{2}$ which induces $\bar{a}_{1}$. Then, if $b \in B$ induces $\bar{a}_{2}$, then $b$ also induces $\bar{a}_{1}$. Thus, in all cases, the 1.t.'s in $V_{n}$ can be induced by elements of $B$. This completes the proof of the theorem below.

Theorem 3.2. Let $A$ be a noncommutative primitive ring, and let $B$ be a subring such that $A / B$ is radical. Then, if $A$ is represented as a dense ring of l.t.'s in a vector space $V$ over a division ring, then $B$ is isomorphic to a dense subring of l.t.'s in $V$.

Corollary. If $A$ is a primitive ring, and if $B$ is a subring such that $A / B$ is radical, then $B$ is a primitive ring.

Proof. If $A$ is noncommutative, then $B$ is primitive by the theorem. If $A$ is commutative, then $A$ is a field, and so is $B$, by [1, Lemma 3].

Note that Theorem 3.2 (together with [1, Theorem A]) is strong enough to imply both Theorems 1.1 and 1.2, after the reduction to the primitive case.

The following was suggested by a remark of the referee:

If $A$ is a semisimple ring, and if $A$ is radical over a subring $B$, then $B$ is semisimple.

Proof. Let $P$ denote the set of all primitive ideals of $A$. Then $\{P \cap B \mid P \in P\}$ is a collection of primitive ideals of $B$. This can be seen as follows: Since $A-P$ is a primitive ring which is radical over the subring $(P+B)-P$, by the corollary, $(P+B)-P$ is a primitive ring, for each $P \in \mathcal{P}$. Now $B-(P \cap B)$, being isomorphic to $(P+B)$ $-P$, is also a primitive ring, that is, $(P \cap B)$ is a primitive ideal of $B$, for each $P \in \mathcal{P}$. Since $\cap_{P \in \mathcal{P}} P=\{0\}$, clearly, $\cap_{P \in \mathcal{P}}(P \cap B)=\{0\}$, and, therefore, $B$ is semisimple. 
4. This section contains an application of Theorem 1.1. Let $A$ be a division ring with center $Z \neq A$; let $B$ be a division subring, $B \neq A$, $B \nsubseteq Z$; let $S^{*}=\{0 \neq a \in S\}$ and $N(S)=\left\{a \in A^{*} \mid a^{-1} S a=S\right\}$, for any division subring $S$ of $A$; and let

$$
N_{0}=\bigcap_{a \in A^{*}} N\left(a^{-1} B a\right) .
$$

Hattori has shown that $N_{0}=Z^{*}$ under the hypothesis that $A$ is finite dimensional over $Z$, and $A \supset B \supset Z$. This result, together with Kaplansky's theorem [7], shows that $A^{*} / N_{0}$ is not a torsion group, when $[A: Z]<\aleph_{0}$. The principal result of my article, On conjugates in division rings, Canad. J. Math. vol. 10 (1958) pp. 374-380, is the following theorem that holds for arbitrary $A$ and $B$ as above: $A^{*} / N_{0}$ is not finite (in fact, $N(B)$ has infinite index in $A^{*}$ ). Whether $A^{*} / N_{0}$ can be torsion for some (necessarily infinite dimensional) $A$ is an open question. If $A^{*} / N_{0}$ is torsion, then Theorem 1.1 shows that the division subring generated by $N_{0}$ coincides with $A$. Aside from this, Theorem 1.1 can be used to obtain the following

TheOREM 4.1. Assume the notation directly above. If $A$ has characteristic $p>0$, then $A^{*} / N_{0}$ is not a $p$-group.

Proof. Assume that $A^{*} / N_{0}$ is a $p$-group. Then, if $0,-1 \neq v \in A$, there exists an exponent $e>0$ such that both $v^{p^{0}}$ and $(v+1)^{p^{*}} \in N$, $N=N(B)$. Then, since both $v^{p^{*}}$ and $v^{p^{*}}+1$ lie in $N$, by Brauer's result (cf., loc. cit., Proposition 3), it follows that:

(*) To each $v \in A$ there corresponds $e>0$ such that $v^{p^{*}} \in B \cup B^{\prime}$ (set union).

(Here, $B^{\prime}$ denotes the centralizer of $B$ in $A$.) This, together with Theorem 1.1, implies that $B \nsubseteq B^{\prime}$ and that $B^{\prime} \Phi B$, since a noncommutative division ring cannot be radical over a proper subring. Since $B$ is thereby not equal to $B \cap B^{\prime}, B$ is itself noncommutative, so that, by applying Theorem 1.1 again, there exists an element $a \in B$ which is not radical over $B \cap B^{\prime}$. Let $v=a+b$, where $b \in B^{\prime}$. By $(*), v^{p^{*}}$ $=a^{p^{*}}+b^{p^{*}} \in B \cup B^{\prime}$ for some $e>0$. Since $a^{p^{*}} \notin B^{\prime}$, necessarily $v^{p^{*}} \in B$, whence $b^{p^{*}} \in B \cap B^{\prime}$. This shows:

$$
\text { Some power } p^{\circ} \text { of each } b \in B^{\prime} \text { lies in } B \text {. }
$$

Now let $v$ be any element of $A$ not radical over $B$. By (*), $v^{p^{*}} \in B^{\prime}$ for some $e>0$. By $(* *), v^{p^{k}} \in B$ for some $k \geqq e$, contrary to the choice of $v$. This contradiction establishes the theorem. 


\section{REFERENCES}

1. Carl Faith, Algebraic division ring extensions, Proc. Amer. Math. Soc. vol. 11 (1960) pp. 43-53.

2. - A structure theory for semialgebraic extensions of division algebras, J. Reine Angew. Math., to appear.

3. I. N. Herstein, $A$ theorem on rings, Canad. J. Math. vol. 5 (1953) pp. 238-241.

4. - Two remarks on the commutativity of rings, Canad. J. Math. vol. 7 (1955) pp. 411-412.

5. Nathan Jacobson, Structure theory for algebraic algebras of bounded degree, Ann. of Math. vol. 46 (1945) pp. 695-707. 1956.

6. - Structure of rings, Amer. Math. Soc. Colloquium Publications, vol. 37,

7. Irving Kaplansky, $A$ theorem on division rings, Canad. J. Math. vol. 3 (1951) pp. 290-292.

8. J. H. M. Wedderburn, A theorem on finite algebras, Trans. Amer. Math. Soc. vol. 6 (1905) pp. 349-352.

The Pennsylvania State University

\section{NOTE ON THE NONVANISHING OF $L(1)$}

S. CHOWLA AND L. J. MORDELL

It is well known that if $\chi(m)$ is a real nonprincipal character $(\bmod k)$, then

$$
L(1)=\sum_{1}^{\infty} \frac{\chi(m)}{m} \neq 0,
$$

and many proofs have been found. We give a very simple proof when $k=p$ an odd prime, in which case $\chi(m)=(m / p)$, the Legendre symbol. This makes it possible to simplify the proof that if $p \nmid a$, then there are infinitely many primes congruent to $a$ modulo $p$. Write

$$
\zeta=e^{2 \pi i / p}, \quad P=\frac{\prod_{n}\left(1-\zeta^{n}\right)}{\prod_{r}\left(1-\zeta^{r}\right)},
$$

where $n$ runs through the quadratic nonresidues of $p$ and $r$ runs

Received by the editors May 2, 1960. 